\title{
Оптимизация аналитической процедуры одновременного хроматографического выделения свинца, меди и цинка
}

\author{
Окунева Т.Г., Стрелецкая М.В., Солошенко Н.Г., Киселева Д.В.
}

Институт геологии и геохимии им. ак.А.Н. Завариџкого УрО РАН, Екатеринбург, okunevatatjana@mail.ru

\begin{abstract}
Аннотация. Исследование отношений радиогенных (свинец) и стабильных (медь и цинк) изотопных отношений может помочь в детерминации источников загрязнений и их миграции в окружающей среде. В данной работе предложена аналитическая процедура одновременного хроматографического выделения свинца, меди и цинка из мультиэлементного стандартного раствора PerkinElmer, стандартного образца почвы SoFC-1 Bioavailability RM (EPA/USGS, Flat Creek, Motana, USA) и образца отложений локальных понижений микрорельефа г. Екатеринбурга. Массовый выход свинца, меди и цинка составил для всех образцов не менее 89, 94 и 71 мас. \% соответственно. Достигнута практически полная очистка меди и цинка от матричных элементов, оказывающих влияние на результаты измерений изотопных отношений. С целью увеличения количественного выхода цинка планируется продолжить исследование в данном направлении.

Ключевые слова: изотопные отношения свинца, меди, цинка; ионообменная хроматография; МС ICPMS; загрязнение окружающей среды.

\section{Optimization of the analytical procedure for the simultaneous chromatographic purification of lead, copper, and zinc}

\section{Okuneva T.G., Streletskaya M.V., Soloshenko N.G., Kiseleva D.V.}

A.N. Zavaritsky Institute of Geology and Geochemistry, Ural Branch of the Russian Academy of Sciences, Ekaterinburg,okunevatatatjana@mail.ru.
\end{abstract}

Abstract. The investigation of multiple radiogenic (lead) and stable (copper and zinc) isotope systems can help to discriminate pollution sources and reveal elemental migration paths in the environment. In this paper, we propose an analytical procedure for the simultaneous chromatographic isolation of lead, copper, and zinc. Standard Multi-Element calibration solution (PerkinElmer, USA), SoFC-1 Bioavailability Reference Material (EPA/USGS, soil from Flat Creek, Motana, USA), as well as the sample of urban sediment from the local microrelief depressions (Ekaterinburg, Russia), were used to obtain elution curves. The mass yield of lead, copper, and zinc for all the samples studied was not less than 89,94 , and $71 \%$, respectively. Almost complete purification of copper and zinc from matrix elements affecting the results of isotopic ratio measurements is achieved. In order to increase the quantitative yield of zinc, it is planned to continue research in this direction.

Keywords: $\mathrm{Pb}, \mathrm{Cu}, \mathrm{Zn}$ isotopes; ion exchange chromatography; MC-ICP-MS, environmental pollution.

\section{Введение}

Загрязнение окружающей среды является глобальной экологической проблемой, оказывающей сильное влияние на здоровье населения. Для контроля качества воздуха, почвы и воды важное значение имеет оценка вклада антропогенных источников потенциально опасных веществ (Vasic et al., 2012; Seleznev et al., 2020; Souto-Oliveira et al., 2018). В последние десятилетия (примерно с начала двухтысячных годов) отношения стабильных изотопов исследовались с целью выявления их потенциала для улучшения понимания источников загрязнений и их миграций в окружающей среде, включая выбросы антропогенных аэрозолей от промышленных, металлургических предприятий и угольных ТЭЦ. Свинец, медь и цинк представляют собой исключительный интерес, поскольку они являются неотъемлемыми компонентами различных искусственных материалов и технологических устройств, произведенных человеком, и их геохимические циклы в значительной степени изменены антропогенной деятельностью.

Измерение изотопных отношений свинца, меди и цинка может быть проведено методом мультиколлекторной масс-спектрометрии с индуктивно-связанной плазмой (MC-ICP-MS) (Albarède et al., 2015). На прецизионность процедуры измерения непосредственное влияние оказывает способ 
учета масс-дискриминации и качество предварительной подготовки образцов (стадия разложения и выделения чистых фракций аналитов). Одним из наиболее известных методов отделения меди и цинка от матричных элементов является их хроматографическое выделение на ионообменной смоле Bio-Rad AG MP-1 (Chapman et al., 2006; Karpova et al., 2019; Стрелецкая и др., 2018; Стрелецкая и др., 2019). В случае определения изотопных отношений меди, цинка и свинца в экологических объектах одновременное выделение чистых фракций аналитов является актуальной задачей.

Цель данной работы заключалась в оптимизации хроматографической процедуры выделения свинца, меди и цинка для последующего изотопного анализа методом MC-ICP-MS.

\section{материалы и методы}

Реагенты, образиьь

Всю химическую подготовку образцов проводили в чистых помещениях (class 1,000 ИСО 6) и ламинарных боксах (класс 100, ИСО 5) Института геологии и геохимии им. А.Н. Заварицкого УрО РАН, Екатеринбург, Россия. Кислоты марки ОСЧ $\left(\mathrm{HCl}, \mathrm{HNO}_{3}\right.$ и $\left.\mathrm{HF}\right)$ предварительно очищали дважды при температуре, не превышающей температуру кипения, в системах очистки (Savillex,

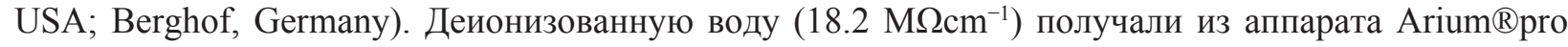
(Sartorius, Germany). Вся посуда, контактирующая с реагентами и образцами, изготовлена из PFA (Savillex, USA) или PTFE (Nalgene, USA). Перед анализом, проводили дополнительную очистку хроматографических колонок, наконечников дозаторов и PFA виал (Savillex $\left.{ }^{\circledR}\right)$. Наконечники и колонки выдерживали в смеси $\mathrm{HCl}: \mathrm{H}_{2} \mathrm{O}(1: 1)$ на горячей плитке в течение суток с последующим ополаскиванием деионизованной водой. Виалы очищали кипячением в смеси $\mathrm{HNO}_{3}: \mathrm{HCl}(1: 3)$ в течение суток с последующим кипячением в деионизованной воде. Для калибровки хроматографических колонок использовали: Multi-Element calibration Standard 3 (PerkinElmer, USA), содержащий 29 элементов, включая $\mathrm{Cu}, \mathrm{Pb}, \mathrm{Zn}$; стандартный образец почвы Bioavailability Reference Material SoFC-1 (EPA/USGS, Flat Creek, Motana, USA); образец отложений локальных понижений микрорельефа г. Екатеринбурга.

\section{Кислотное разложение}

Навески стандартного образца и пробы весом 0.008 г отбирали в PFA виалы, добавляли 3 см$^{3}$ $\mathrm{HNO}_{3}$ и $1 \mathrm{~cm}^{3} \mathrm{HF}$ (конц). Виалы закрывали и выдерживали в сушильном шкафу при $120^{\circ} \mathrm{C}$ в течение 3 дней. После выпаривания досуха добавляли смесь $1 \mathrm{~cm}^{3} \mathrm{HNO}_{3}$ и $3 \mathrm{~cm}^{3} \mathrm{HCl}$ (конц) и снова выпаривали. Затем остаток растворяли в $4 \mathrm{~cm}^{3} \mathrm{HCl}$ конц. и повторяли процедуру выпаривания до сухого остатка. Далее к остатку добавляли 1 см³ $^{3} \mathrm{M} \mathrm{HCl}$ и центрифугировали образец при 6000 об/мин в течение 15 мин.

\section{Хроматографическое выделение меди, цинка и свинца}

Для получения фракции меди, свинца и цинка применяли модифицированный хроматографический метод, приведенный в (Souto-Oliveira et al., 2019). Смолу Bio-Rad AG MP-1 (100-200 меш) загружали в предварительно очищенные полипропиленовые колонки (Bio-Rad®) с двумя $35 \mu \mathrm{m} P \mathrm{E}$ фриттами для удержания смолы. Параметры слоя смолы были следующими: $\mathrm{D}=0.8 \mathrm{~cm}, \mathrm{~h}=1.3 \mathrm{~cm}$, $\mathrm{V}=0.6 \mathrm{~cm}^{3}$. На рисунке 1 приведен протокол процедуры последовательного разделения свинца, меди и цинка.

Протокол элюирования включал стадию подготовки смолы в $2 \mathrm{~cm}^{3} 0.5 \mathrm{M} \mathrm{HNO}_{3}, 2 \mathrm{~cm}^{3}$ деионизованной воды, $4 \mathrm{~cm}^{3} 7 \mathrm{M} \mathrm{HCl}$. Pb и Сu элюировали в $7 \mathrm{M} \mathrm{HCl}, \mathrm{Zn}$ в $0.5 \mathrm{M} \mathrm{HNO}_{3}$. Массовый выход свинца, меди и цинка оценивали измерением их концентраций в мультиэлементном стандарте (Multi-element calibration Standard), стандартном образце и пробе отложений локальных понижений микрорельефа до хроматографического разделения и при построении кривых элюирования. Для измерения концентраций элементов применяли квадрупольный масс-спектрометр NexION 300S (PerkinElmer, USA). Измеренные исходные содержания свинца, меди и цинка во всех образцах приведены в таблице 1. 


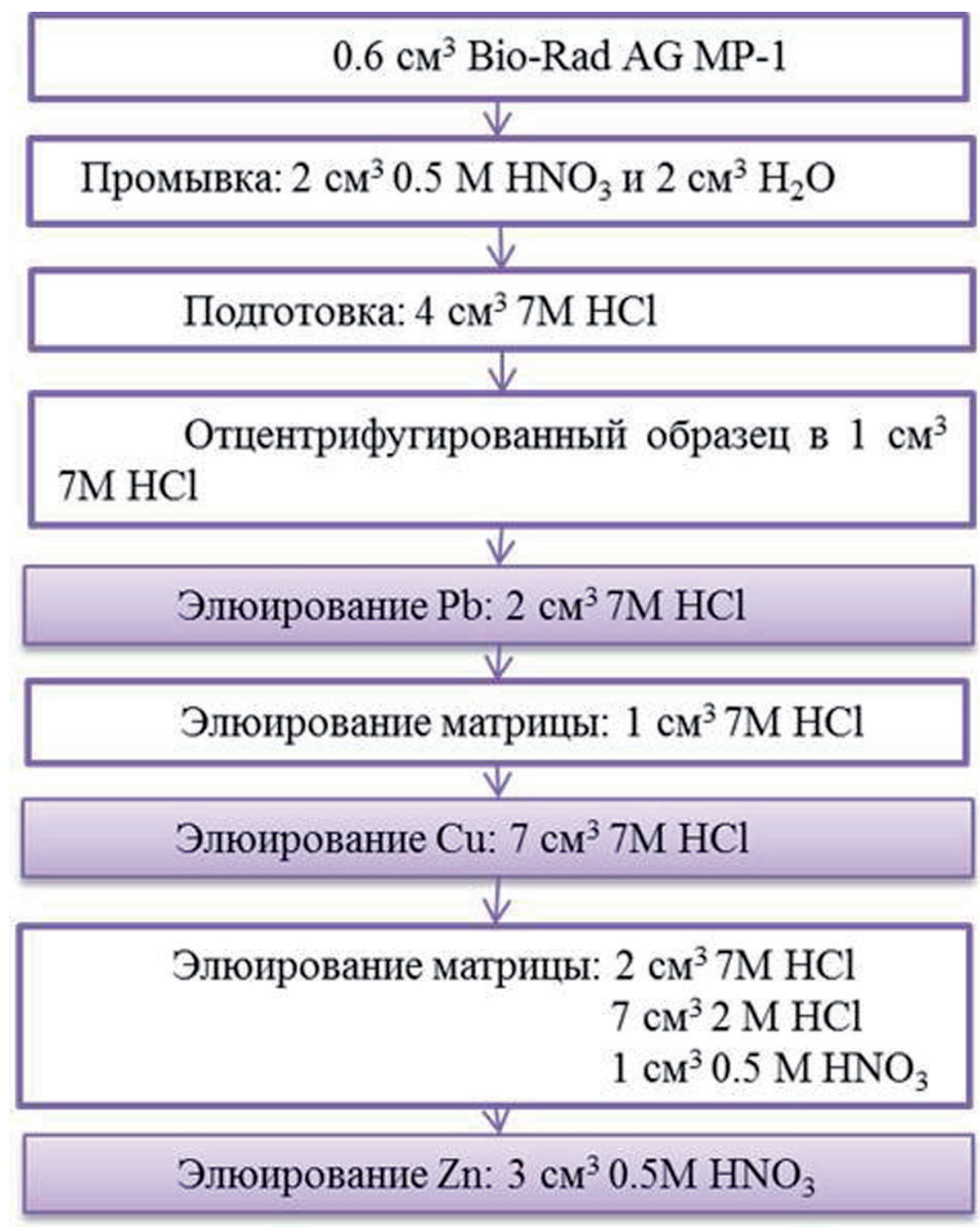

Рис. 1. Схема хроматографического выделения свинца, меди и цинка на смоле Bio-Rad AG MP-1.

Fig. 1. Scheme of $\mathrm{Pb}, \mathrm{Cu}, \mathrm{Zn}$ chromatographic separation using Bio-Rad AG MP-1 resin.

Таблица 1. Содержание свинца, меди и цинка в исследуемых образцах.

Table 1. Concentration of lead, zinc, and copper in the studied samples.

\begin{tabular}{|l|c|c|c|}
\hline & $\mathrm{C}_{\mathrm{pb}}, \mathrm{ppm}$ & $\mathrm{C}_{\mathrm{Cu}}, \mathrm{ppm}$ & $\mathrm{C}_{\mathrm{Zn}}, \mathrm{ppm}$ \\
\hline Multi-element calibration Standard & 1.3 & 1.4 & 1.6 \\
\hline Bioavailability Reference Material SoFC-1 & 22 & 3206 & 1283 \\
\hline Образец отложений локальных понижений микрорельефа г. Екатеринбург & 136 & 333 & 116 \\
\hline
\end{tabular}

\section{Результаты и обсуждения}

Кривые элюирования, полученные для мультиэлементного раствора, стандартного образца и пробы отложений локальных понижений микрорельефа г. Екатеринбург, изображены на рисунке 2. Как видно из рисунка, удается успешно последовательно выделить фракции свинца, меди и цинка. На первой стадии элюируется свинец, одновременно с матричными элементами $(\mathrm{Sr}, \mathrm{Ni}, \mathrm{Mn}$, $\mathrm{Cr}, \mathrm{V}, \mathrm{Al}, \mathrm{Mg}, \mathrm{Na}$ ). Поэтому необходимо дополнительно доочистить аналит от матрицы в соответствии с документом «Методика измерений изотопных отношений свинца в горных породах и 

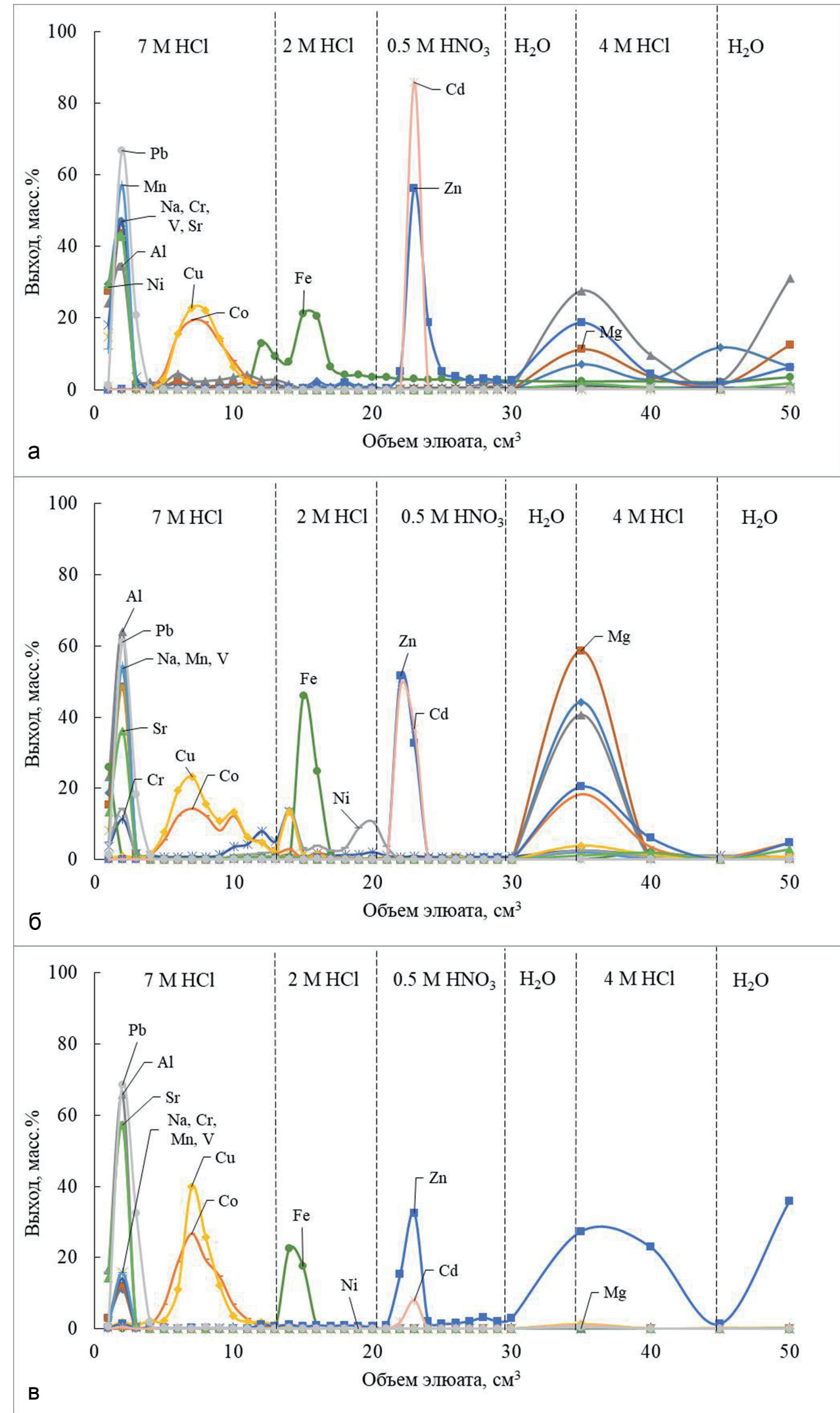

Рис. 2. Кривые элюирования мультиэлементного стандартного раствора (а), стандартного образца (б) и пробы (в). Концентрации $\mathrm{Pb}, \mathrm{Cu}, \mathrm{Zn}$ и матричных элементов ( $\mathrm{Na}, \mathrm{Mg}, \mathrm{Mn}, \mathrm{Al}, \mathrm{V}, \mathrm{Cr}, \mathrm{Ni}, \mathrm{Fe}, \mathrm{Sr}, \mathrm{Cu}, \mathrm{Cd}, \mathrm{Co}$ ) измеряли в элюате с шагом в 1 и $5 \mathrm{~cm}^{3}$.

Fig. 2. Elution curves for multi-element standard (a), reference material (б), and sample (в). Concentrations of $\mathrm{Pb}$, $\mathrm{Cu}, \mathrm{Zn}$, and matrix elements ( $\mathrm{Na}, \mathrm{Mg}, \mathrm{Mn}, \mathrm{Al}, \mathrm{V}, \mathrm{Cr}, \mathrm{Ni}, \mathrm{Fe}, \mathrm{Sr}, \mathrm{Cu}, \mathrm{Cd}, \mathrm{Co}$ ) were obtained for 1.0 and $5 \mathrm{~cm}^{3}$ eluate portions. 
минералах методом масс-спектрометрии с индуктивно связанной плазмой с использованием массспектрометра высокого разрешения Neptune Plus» № 88-16360-009-2014, разработанным в ИГГ УрО РАН. В элюате меди и цинка присутствует некоторое количество кобальта и кадмия - 84 и 86 мас. \% соответственно. Данные элементы не оказывают изобарных и полиатомных наложений на аналиты (Mason et al., 2004) и не влияют на измерение их изотопных отношений методом MC ICP-MS. Средний массовый выход свинца, меди и цинка в собираемых фракциях для всех трех исследуемых образцов составил не менее 89, 94 и 71 мас. \% соответственно. Из кривых элюирования видно, что около 30 \% цинка элюируется в деионизованной воде при регенеративной промывке колонки, поэтому дальнейшие наши исследования будут направлены на оптимизацию условий количественного выделения цинка с использованием данной хроматографической системы.

\section{Вывод}

В данной работе представлена аналитическая процедура хроматографической очистки свинца, меди и цинка для последующего изотопного анализа на мультиколлекторном масс-спектрометре с индуктивно-связанной плазмой. В ходе проведения исследований удалось успешно последовательно выделить аналиты. При этом, необходима дополнительная очистка фракции свинца с использованием выделения на анионнообменной смоле AG1-X8. Нами была достигнута практически полная очистка меди и цинка от матричных элементов, оказывающих влияние на результаты измерений изотопных отношений. Целью наших дальнейших исследований является оптимизация хроматографических условий выделения цинка.

Работа выполнена в ЦКП «Геоаналитик» и поддержана грантом РНФ №16-17-10283.

\section{Литература}

1. Стрелецкая М.В., Киселева Д.В., Зайцева М.В., Белогуб Е.В. Изотопный анализ меди в медьсодержащих сульфидах с использованием мультиколлекторной масс-спектрометрии с индуктивно связанной плазмой // Металлогения древних и современных океанов. 2018. Т. 24. С. 261-265.

2. Стрелецкая М.В., Червяковская М.В., Окунева Т.Г., Киселева Д.В. Применение стандартных образцов BHVO-2, AGV-2, BCR-2 для валидации методики определения изотопных отношений цинка в природных объектах // Уральская минералогическая школа. 2019. Т. 25. С. 169-175.

3. Albarède F., Albalat E., Télouk P. Instrumental isotope fractionation in multiplecollector ICP-MS // Journal of Analytical Atomic Spectrometry. 2015. V. 30. P. 1736-1742. DOI:10.1039/C5JA00188A.

4. Chapman J.B., Mason T.F.D., Weiss D.J., Coles B.J., Wilkinson J.J. Chemical separation and isotopic variations of $\mathrm{Cu}$ and $\mathrm{Zn}$ from five geological reference materials // Geostandards and Geoanalatycal Research. 2006. V. 30. P. 5-16. DOI:10.1111/j.1751-908X.2006.tb00907.x.

5. Karpova S.V., Kiseleva D.V., Chervyakovskaya M.V., Streletskaya M.V., Shagalov E.S., Bogdanov S.V., Tkachev V.V., Yuminov A.M. and Ankushev M.N. Copper isotope ratios in Cis-Urals coppersandstones and products of their processingas a tool for uncovering the Bronze Agesmelting activities // AIP Conference Proceedings. 2019. V. 2174. P. 020221. https://doi.org/10.1063/1.5134372.

6. Mason T.F.D., Weiss D.J., Horstwood M., Parrish R.R., Russell S.S., Mullane E. \& Coles B.J. (2004). Highprecision $\mathrm{Cu}$ and $\mathrm{Zn}$ isotope analysis by plasma source mass spectrometry. Journal of Analytical Atomic Spectrometry, 19(2), 209. doi:10.1039/b306958c.

7. Seleznev A.A., Yarmoshenko I.V., Malinovsky G.P. Urban geochemical changes and pollution with potentially harmful elements in seven Russian cities // Scientific Reports. 2020. 10. 1668. DOI:10.1038/s41598-020-58434-4.

8. Souto-Oliveira C.E., Babinski M., Araújo D.F., Andrade M.F. Multi-isotopic fingerprints ( $\mathrm{Pb}, \mathrm{Zn}, \mathrm{Cu})$ applied for urban aerosol source apportionment and discrimination // Science of The Total Environment.2018. V. 626. P. 1350-1366. DOI:10.1016/j.scitotenv.2018.01.192.

9. Souto-Oliveira C.E., Babinski M., Araújo D.F., Weiss D.J, Ruiza. I.R. Multi-isotope approach of Pb, $\mathrm{Cu}$ and $\mathrm{Zn}$ in urban aerosols and anthropogenic sources improves tracing of the atmospheric pollutant sources in megacities // Atmospheric Environmental. 2019. V.198. P. 427-437. DOI: 10.1016/j.atmosenv.2018.11.007.

10. Vasic M.V., Mihailovic A., Kozmidis-Luburic U., Nemes T., Ninkov J., Zeremski-Skoric T., Antic B. Metal Contamination of Short-Term Snow Cover near Urban Crossroads: Correlation Analysis of Metal Content and Fine Particles Distribution // Chemosphere. 2012. V. 85. P. 585-592. DOI: 10.1016/j.chemosphere.2011.10.023 